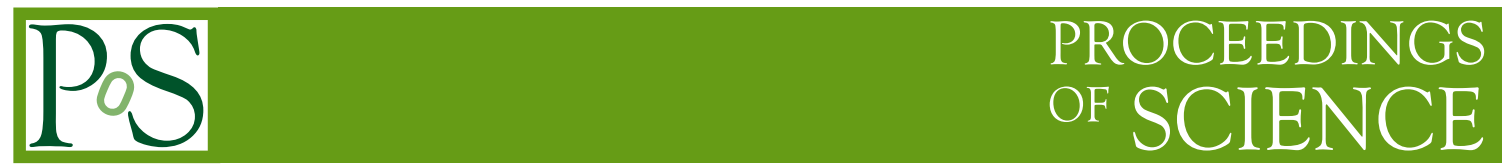

\title{
Dark Matter Theory
}

\author{
Alessandro Strumia* \\ Dipartimento di Fisica dell'Università di Pisa and INFN, Italia \\ E-mail: astrumialdf.unipi.it
}

We summarize the status of Dark Matter, focussing on recent developments, mostly prompted by the cosmic-ray excess observed by PAMELA and FERMI.

European Physical Society Europhysics Conference on High Energy Physics, EPS-HEP 2009,

July 16 - 222009

Krakow, Poland

${ }^{*}$ Speaker. 


\section{Introduction}

Cosmology and astrophysics established that DM exists, but so far could see DM only via its gravitational interactions, weighting the total DM amount: $\Omega_{\mathrm{DM}} h^{2}=0.110 \pm 0.005$ [1]. This is all the positive information we have so far on DM. Various bounds tell that anything that behaves as collision-less dust can be DM: from an oscillating ultra-light scalar to a neutral new particle. In the latter case the DM cosmological abundance could be determined by thermal freeze-out within standard big-bang: in such a case the observed DM abundance is reproduced for a well defined value of the DM annihilation cross section:

$$
\sigma v \approx \frac{1}{T_{\text {now }} M_{\mathrm{Pl}}} \approx 3 \cdot 10^{-26} \frac{\mathrm{cm}^{3}}{\mathrm{sec}}=\frac{0.0025}{\mathrm{TeV}^{2}} \quad \text { at velocity } v \sim 0.2
$$

where numerical values include order one factors. This $\sigma v$ is typical of weak scale particles. If DM is the relic of a weak scale particle, we can soon discover in the following three ways:
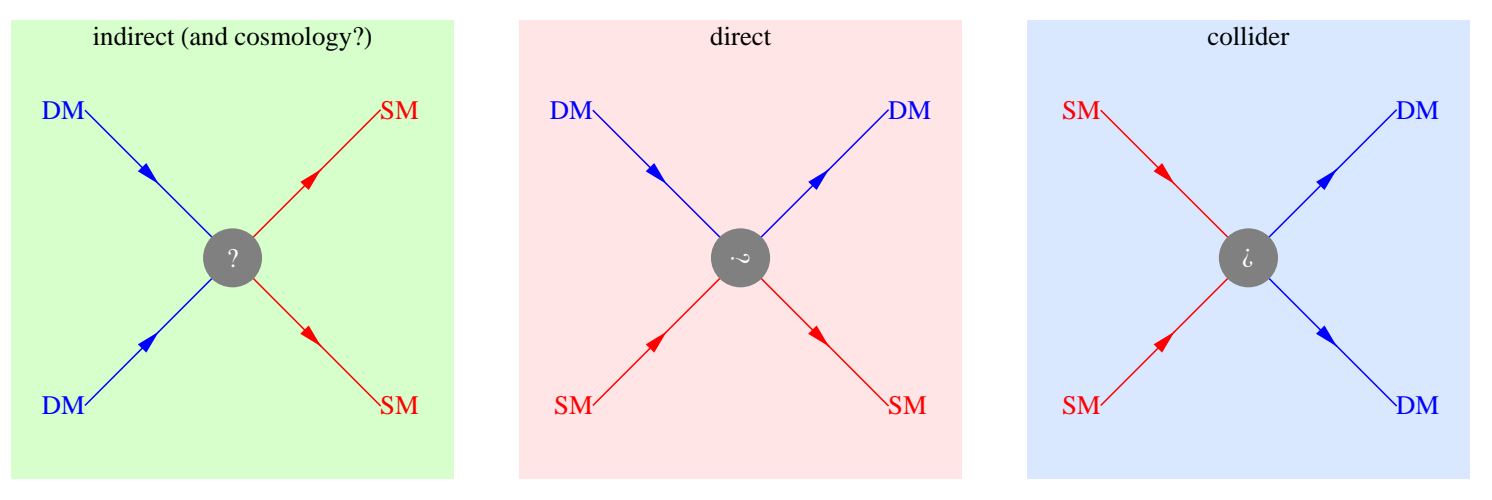

i.e. i) we hope to see end-products of astrophysical DM annihilations in cosmic rays; ii) we hope to see DM scattering on normal matter; iii) we hope to produce DM at LHC. As we have no idea of which SM particle is involved in DM couplings, we do not know which search is better. In the next sections we summarize the various possibilities.

\section{Cosmology and colliders}

Eq. (1.1) suggests DM at the weak scale: the key question is if DM is expected to be light enough to be produced at the LHC. As a typical cross section is $\sigma \sim g^{4} / 4 \pi M^{2}$, heavier DM masses are obtained for larger DM couplings $g$. Many theories of Dark Matter, such as the MSSM, are compatible with a wide range of DM couplings, and consequently with a wide range of DM masses $M$. In order to predict $M$, we need to predict $g$ : we assume that DM fills one weak multiplet that only has gauge interactions [13]. The following table shows in the few main cases the predicted 
DM masses (taking into account co-annihilations and Sommerfeld enhancements):

\begin{tabular}{|cc|ccccc|}
\hline $\begin{array}{c}\text { Quantum numbers } \\
\mathrm{SU}(2)_{\mathrm{L}} \mathrm{U}(1)_{Y} \text { Spin }\end{array}$ & $\begin{array}{c}\text { nick- } \\
\text { name }\end{array}$ & $\begin{array}{c}\text { DM mass } \\
\text { in TeV }\end{array}$ & $\begin{array}{c}\text { Events at LHC } \\
\int \mathscr{L} d t=100 / \mathrm{fb}\end{array}$ & $\begin{array}{c}\sigma_{\mathrm{SI}} \text { in } \\
10^{-45} \mathrm{~cm}^{2}\end{array}$ \\
\hline \hline 2 & $1 / 2$ & 0 & sneutrino & 0.54 & $\sim 400$ & 0.3 \\
2 & $1 / 2$ & $1 / 2$ & higgsino & 1.2 & $\sim 200$ & 0.3 \\
\hline 3 & 0 & 0 & - & 2.5 & $\sim 1$ & 1.3 \\
3 & 0 & $1 / 2$ & wino & 2.7 & $\sim 2$ & 1.3 \\
\hline 5 & 0 & $1 / 2$ & stable & 9.6 & 0 & 12 \\
\hline
\end{tabular}

The main message is that the typical DM mass $M$ is around or above $1 \mathrm{TeV}$. This is bad for two reasons: DM heavier than a TeV would be hardly discoverable at LHC (the low event rate can get lost in the backgrounds) and would not suggest a clean connection with the hierarchy problem of the electroweak scale. DM lighter than a TeV annihilates too much leaving a too low abundance, unless one or more of the following possibilities are realized:

- If DM is lighter than the $W$, the too large $\sigma\left(\mathrm{DM} \mathrm{DM} \rightarrow W^{+} W^{-}\right)$gets kinematically suppressed. Such a possibility, realized e.g. in 'intert Higgs doublet' models is not fully excluded by LEP searches.

- If DM is a mixture between a gauge neutral singlet with gauge charged multiplet(s), one can reduce DM gauge couplings allowing any DM mass lighter than in table (2.1). This is possible within the MSSM, where a DM neutralino is an arbitrary mixing between bino, higgsino and wino.

- DM could be a singlet which only has some non-gauge interactions. This was the best possibility within the MSSM, with DM as a bino with supergauge Yukawa interactions to leptons and sleptons, but it got mostly excluded by LEP searches. The observed $\Omega_{\mathrm{DM}}$ is obtained only if sleptons are just above their LEP bound. (Another possibility allowed within the MSSM is that $\sigma$ is resonantly enhanced if the pseudo-scalar Higgs happens to have a mass very close to $2 M$ ).

- Within the MSSM, the main channel for DM production at the LHC is not direct but indirect production of gluinos that cascade decay down to the DM LSP. If DM is the gravitino, the long-lived NLSP can give clean extra signals.

\section{Direct searches}

Experiments searched for DM elastic scatterings on a variety of nuclei; the main parameter used to compare their data with theory is the spin-independent DM cross section on an average nucleon, $\sigma_{\mathrm{SI}}$, presently constrained to be $\sigma_{\mathrm{SI}} \lesssim 10^{-43} \mathrm{~cm}^{2}$ for $M \sim 100 \mathrm{GeV}$. Weaker bounds apply if DM is heavier or lighter. 
The following Feynman diagrams illustrate three possible sources of DM/nucleon scattering and their typical $\sigma_{\mathrm{SI}}: Z$-exchange, higgs exchange, loop mediation.

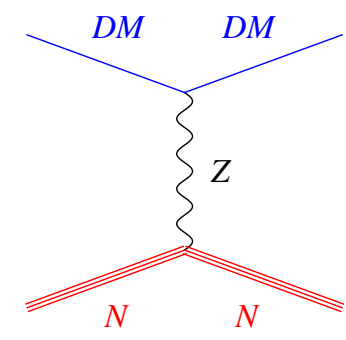

tree, vector

$$
\sigma_{\mathrm{SI}} \approx \frac{\alpha^{2} m_{N}^{2}}{M_{Z}^{4}}
$$

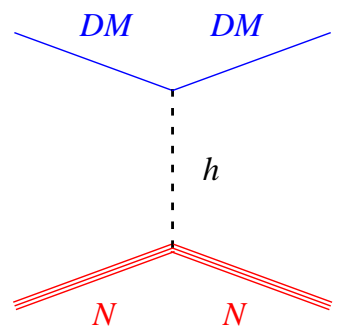

tree, scalar

$\sigma_{\mathrm{SI}} \approx \frac{\alpha^{2} m_{N}^{4}}{M_{h}^{6}}$

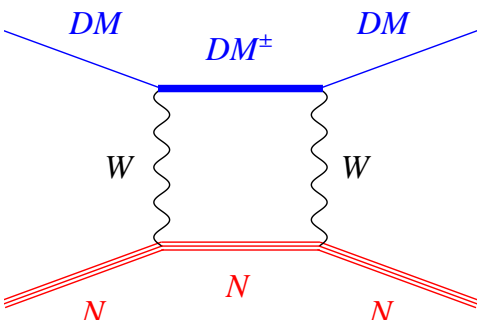

$$
\sigma_{\text {SI }} \approx \frac{\alpha^{4} m_{N}^{4}}{M_{W}^{6}}
$$

$Z$-exchange give a $\sigma_{\mathrm{SI}}$ comparable to $\sigma$ in eq. (1.1), which is excluded by a few orders of magnitude, unless the DM coupling to the $Z$ is correspondingly smaller than $g_{2}$. This naturally happens if DM is a real particle (a real scalar or a Majorana fermion like the neutralino).

The Higgs exchange effect is suppressed by the nucleon mass, but still gives a $\sigma_{\mathrm{SI}}$ somewhat above the experimental bounds unless the DM coupling to the Higgs is somewhat smaller than $g_{2}$.

The loop effect, present if DM lies in a weak multiplet with charged heavier components, is within the sensitivity of planned experiments.

The positive signal claimed by the DAMA experiment is compatible with null results of all other experiments only if DM has unexpected properties, such as a form factor in DM $N$ scattering [3] or an inelastic DM $N \rightarrow \mathrm{DM}^{\prime} N^{\prime}$ with mass difference around $100 \mathrm{keV}$, the DM kinetic energy in our galaxy [2]. These possibilities will be soon confirmed or excluded by running experiments, and appear already disfavored by present data.

\section{Indirect searches}

DM annihilations or decays around the Milky Way can produce SM particles that decay into $e^{ \pm}, p, \bar{p}, \gamma, \bar{d}$, producing an excess in their cosmic ray fluxes.

This topic attracted interest because recently the PAMELA experiment [4] observed an unexpected rise with energy of the $e^{+} /\left(e^{+}+e^{-}\right)$fraction in cosmic rays, suggesting the existence of a new positron component. The sharp rise suggests that the new component may be visible also in the $e^{-}+e^{+}$spectrum: although the peak hinted by previous ATIC data [5] is not confirmed, the FERMI [6] and HESS [7] observations still demonstrate a deviation from the naive power-law spectrum, indicating an excess compared to conventional background predictions of cosmic ray fluxes at the Earth. The excess could be due to a new astrophysical component, such as a nearby pulsar, or could be an experimental mistake ( $p$ contamination in PAMELA, etc.). We here focus on 
DM with $M=3 . \mathrm{TeV}$ that annihilates into $\tau^{+} \tau^{-}$with $\sigma v=2.0 \times 10^{-22} \mathrm{~cm}^{3} / \mathrm{s}$
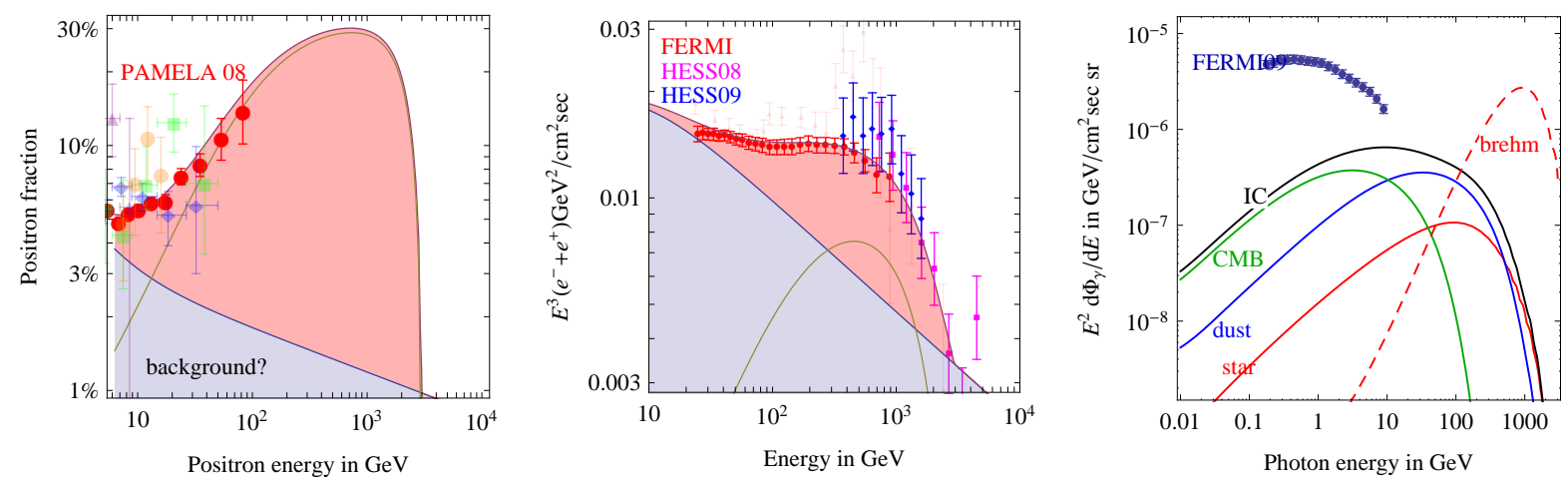

Figure 1: Sample DM fits. We consider DM annihilations into $\tau^{+} \tau^{-}$with MED diffusion [8] and the isothermal DM profile: all good fits are very similar. Left: the positron fraction compared with the PAMELA excess. Middle: the $e^{+}+e^{-}$flux compared with the FERMI and HESS data. Right: the DM contribution to the diffuse photon energy spectra produced by bremsstrahlung (dashed red curve) and Inverse Compton (black thick line); we also separately show the 3 IC components from star-light (red), CMB (green), dust (blue).

the most interesting possibility: the excess could be the first manifestation of Dark Matter, rather than a new background to Dark Matter searches.

Fig. 1 shows the PAMELA (left) and FERMI, HESS (middle) data together with a possible DM fit.

\subsection{The $e^{+}$PAMELA excess}

As directions of charged comic rays get randomized by galactic magnetic fields, the information lies in the energy spectra. Dark Matter could manifest as an excess in the rarer positrons or anti-protons. Observations so far have been made only below $100 \mathrm{GeV}$ : the experimental difficulty is bringing above the atmosphere a large enough calorimeter with a spectrometer able of discriminating the sign of the charge. Furthermore, cosmic ray fluxes roughly decrease as $E^{-3}$.

Observations below $\sim 10 \mathrm{GeV}$ are affected by the solar activity and thereby provide essentially no information on the underlying particle physics.

According to standard astrophysics, the positron/electron fraction above $10 \mathrm{GeV}$ should decrease with energy, while PAMELA finds the steep increase in fig. 1a, signaling a new component. As the relevant DM annihilations into pairs of SM particles are non-relativistic, given any channel the energy spectra of the final stable $e^{+}, \bar{p}$ can be computed in a model-independent way.

PAMELA data suggest two classes of DM interpretations for the excess: a) DM that annihilates into pairs of charged leptons, of $W$ or of $Z$, with any DM mass above about $100 \mathrm{GeV}$; b) DM that annihilates into pairs of quarks or higgs, but only if $\mathrm{DM}$ is heavier than a few $\mathrm{TeV}$, as these channels give a soft $e^{+}$spectrum. 
The result does not depend much on the unknown DM density profile in our galaxy, $\rho(r)$, nor on the typical galactic DM velocity $\left(v \sim 10^{-3}\right.$ comparable to the escape velocity from the Milky Way) nor on the $e^{ \pm}$propagation model in the turbulent galactic magnetic fields.

The $\sigma v$ at $v \approx 10^{-3}$ needed to fit PAMELA grows with $M$ and typically is a few order of magnitude larger than the value suggested by cosmology of eq. (1.1). Non-relativistic DM annihilations should be dominated by the $s$-wave, such that $\sigma v$ does not depend on $v$, and PAMELA is not compatible with standard cosmology.

Various ways out have been proposed, such as a non-standard cosmologies or a DM clump close to us. A possible particle-physics way of reconciling PAMELA with standard cosmology is $s$-channel DM annihilations mediated by a narrow particle with a mass very close to $2 M$, such that astrophysical annihilations $\left(v \sim 10^{-3}\right)$ can be more resonantly enhanced than cosmological annihilations $(v \sim 0.2)[9,11]$. More interestingly, if DM is charged under a vector with mass $m$ much lighter than DM, the resulting attractive long-range force between pairs of DM particles enhances their annihilation cross section: $\sigma v$ would grow as $1 / v$ for small velocities down to $v \gtrsim m / M$, making PAMELA compatible with standard cosmology [9]. This phenomenon is fully analogous to the QED Sommerfeld enhancement of processes like $\sigma\left(\mu^{+} \mu^{-} \rightarrow e^{+} e^{-}\right)$, and analogous to the classical enhancement of the probability of falling into the sun in view of its long range attractive gravity. In the DM case, the lighter vector could be the $W$ [12], if DM has a multi-TeV mass: in such a case $\mathrm{DM} \mathrm{DM} \rightarrow W^{+} W^{-}$must be one of the main DM annihilation channels [13].

\subsection{The $\bar{p}$ PAMELA observations}

Adding to the data-set the PAMELA [16] observation of a $\bar{p} / p$ fraction compatible with the astrophysical background restricts the range of possible DM interpretations for the $e^{+}$excess, giving another hint in favor of lepto-philic DM $[9,10]$. Indeed DM annihilations into $e, \mu, \tau$ leptons do not give any $\bar{p}$ excess, as leptons do not decay into protons. On the contrary all other DM channels are significantly constrained: trusting the available astrophysical models for $\bar{p}$ propagation and for the $\bar{p}$ background ${ }^{1}$, these channels are compatible with PAMELA $\bar{p}$ data only if $M \gtrsim 10 \mathrm{TeV}[9,17]$. Indeed let us consider for example the $\mathrm{DM} \mathrm{DM} \rightarrow W^{+} W^{-}$channel. A $W$ at rest produces (anti)protons with $E_{p}>m_{p}$, so a $W$ with energy equal to the DM mass $M$ produces protons with $E_{p}>m_{p} M / M_{W}$, which is above the energy range observed by PAMELA if $M$ is heavy enough. (Final State Radiation produces some protons with lower energy). Thereby DM models leave open the possibility that the $e^{+}$excess is accompanied by a $\bar{p}$ excess, but only above 100 $\mathrm{GeV}$.

\subsection{The $e^{+}+e^{-}$ATIC, FERMI and HESS observations}

These experiments provide the best measurements of the electron plus positron cosmic ray spectrum, although ATIC was designed to observe nuclei, and FERMI and HESS to observe photons. The growing excess in the positron fraction observed by PAMELA below $100 \mathrm{GeV}$ could become of order unity at $\mathrm{TeV}$ energies, so that it is interesting to consider measurements of the $e^{+}+e^{-}$cosmic ray spectrum, made by calorimeters that cannot discriminate the $e^{ \pm}$charge. (We

\footnotetext{
${ }^{1} \bar{p}$ backgrounds are predicted with a plausible $\pm 20 \%$ uncertainty. The $\bar{p}$ DM excess can be suppressed if the diffusion zone is small, e.g. if it extends away from the galactic plane for only $1 \mathrm{kpc}$, as in the so-called MIN possibility.
} 
recall that $e^{ \pm}$in matter shower in an exponential way, so that calorimeters become more precise and easily reach higher energies, where spectrometers see all tracks as quasi-straight becoming less precise and eventually useless).

One year ago the ATIC balloon had the best measurement, and its data showed a peak around $700 \mathrm{GeV}$. The FERMI experiment now provides the first high-statistics measurement of the $e^{+}+e^{-}$ spectrum, which do not confirm the ATIC peak, but still indicate an $e^{+}+e^{-}$excess. Indeed the $e^{+}+e^{-}$spectrum is harder than what expected, and the data indicate two spectral features in it, suggesting that the excess appears around $100 \mathrm{GeV}$ and terminates around $1 \mathrm{TeV}$. These features are clearly larger than the statistical errors and it seems unlikely that they could be due to systematic uncertainties, although only the FERMI collaboration can answer the crucial question: are these features really there? HESS observations around and above $1 \mathrm{TeV}$ independently indicate the termination of the excess. The take-over of a new component around $100 \mathrm{GeV}$ should become clearly visible once FERMI data at lower energies will be published.

If both features are real, DM annihilations into $\tau^{+} \tau^{-}$and $\mu^{+} \mu^{-}$can fit the PAMELA, FERMI and HESS data [18].

Interesting theories are constructed adding to the SM a DM particle charged under a new light 'dark vector' $V$, obtaining Sommerfeld-enhanced DM DM $\rightarrow V V$ annihilations. If the vectors is lighter than the proton, it can only decay into the lighter leptons $e, \mu$ and possibly into $\pi$ 's: this scenario nicely explains why DM could annihilate only into leptons [14]. Models where DM annihilations give 4 leptons with a smooth energy spectrum can fit all the data [18]. New vectors with $\mathrm{GeV}$-scale mass mixed with photons can be searched for in low-energy experiments, especially in dedicated electron beam-dump experiments [15].

Models where DM is lighter than about a $\mathrm{TeV}$ (such as a $200 \mathrm{GeV}$ supersymmetric wino [9, 19]) can no longer produce the PAMELA excess, as it would terminate below $1 \mathrm{TeV}$ where FERMI data show a smooth spectrum. ${ }^{2}$ Thereby DM predicts that the $e^{+}$fraction will continue to grow at higher energy as in fig. 1.

\section{4 $\gamma$ and $v$ observations}

The DM DM $\rightarrow \ell^{+} \ell^{-}$interpretations of the $e^{ \pm}$excess predict related excesses in $\gamma$ and $v$ fluxes. Indeed neutrinos are unavoidably generated by $\mu$ and $\tau$ decays, and $\gamma$ 's are unavoidably generated by 3 different processes:

1. Bremsstrahlung from $\ell^{ \pm}$. This gives $\gamma$ with the largest $E_{\gamma} \sim M$, probed by HESS. However the $\gamma$ energy spectrum significantly depends on the DM annihilation mode: modes involving $\tau$ (that decay into $\pi^{0} \rightarrow 2 \gamma$ ) give the largest $\gamma$ yield, while models involving neutral light vectors give the smallest $\gamma$ yield.

2. Inverse Compton: $e^{ \pm}$scatterings on the galactic ambient light (CMB and star light, partially rescattered by dust) give rise to $e^{ \pm} \gamma \rightarrow e^{ \pm} \gamma^{\prime}$ with $E_{\gamma^{\prime}} \sim E_{\gamma}\left(E_{e} / m_{e}\right)^{2} \sim 10 \mathrm{GeV}$, being probed by FERMI. The resulting $e^{ \pm}$energy loss is proportional to the energy density $u_{\gamma}$ in $\gamma$.

\footnotetext{
${ }^{2}$ We do not consider the possibility of a DM component that terminates compensated by a new astrophysical component that grows.
} 

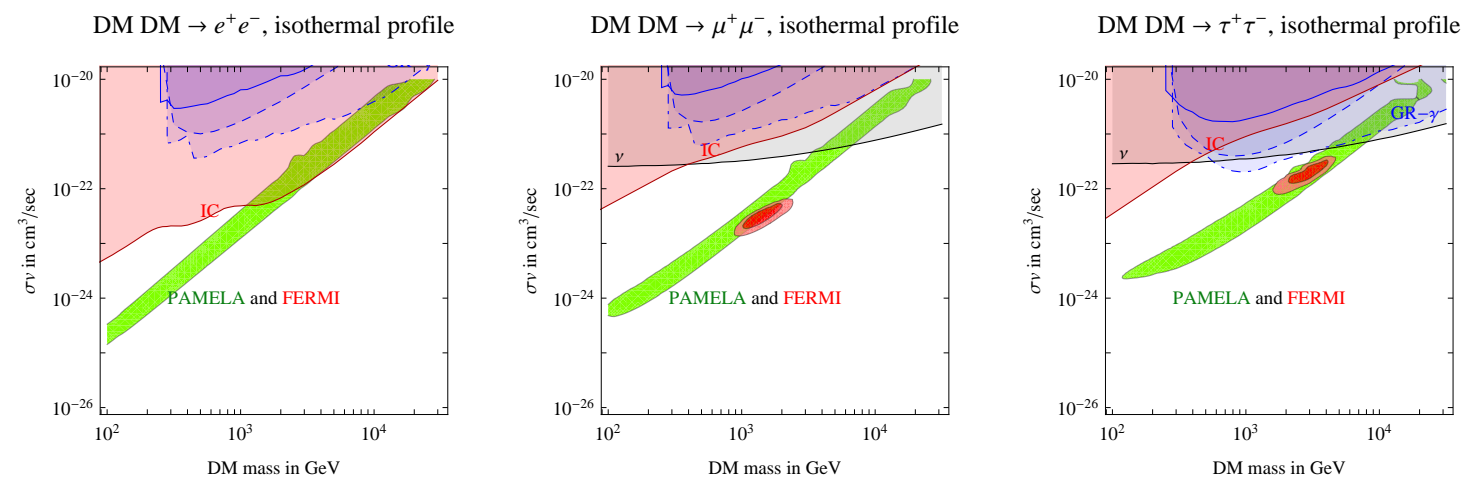

Figure 2: We compare the region favored by PAMELA (green bands) and by PAMELA, FERMI and HESS observations (red ellipses) with $\gamma$ observations of the Galatic Center (blue continuous line), of the Galactic Ridge (blue dot-dashed), of spherical dwarfes (blue dashed), with neutrino data, with FERMI observations in the ' $10^{\circ} \div 20^{\circ}$ ' region.

3. Synchrotron: $e^{ \pm}$diffuse in the galactic magnetic fields, radiating $\gamma$ at radio-frequencies, $E_{\gamma} \sim$ $10^{-6} \mathrm{eV}$, probed by radio-observations: DAVIES, WMAP. The resulting $e^{ \pm}$energy loss is proportional to the energy density $u_{B}=B^{2} / 2$ in magnetic fields.

As it is believed that $u_{\gamma}(\vec{x})$ is about one order of magnitude larger than $u_{B}(\vec{x})$, Inverse Compton is dominant, and it can be reliably computed as essentially all the $e^{ \pm}$energy goes into IC, irrespectively of the precise value of $u_{\gamma}$.

In fig. 2 (from [18]) we compare the values of $\sigma v$ and $M$ suggested by the $e^{ \pm}$excesses with the various $\gamma$ and $v$ bounds, computed imposing that the DM $\gamma$ [20] and $v$ [21] fluxes do not exceed the various observations by more than $3 \sigma$.

We needed to consider the quasi-constant 'isothermal' DM density profile because otherwise, had we assumed the Einasto or NFW profiles favored by $N$-body DM simulations, the DM density around the Galactic Center would have been so large that various bounds would be violated. Interpretations of the $e^{ \pm}$excesses in terms of DM DM $\rightarrow \ell^{+} \ell^{-}$annihilations are thereby excluded, if $N$-body simulations reliably predict the DM density profile.

Models where DM annihilates into two light vectors that decay into lepton pairs (giving rise to $4 \ell$ states) are less constrained: the $\gamma$ FSR flux is roughly reduced by a factor $\ln \left(m / m_{\ell}\right) / \ln \left(M / m_{\ell}\right)$. Despite that, a DM density profile more constant than what suggested by $N$-body simulations remains again needed. If the light vectors have a long kpc-scale life-time, the consequent smoothing of the $e^{ \pm}, \gamma, v$ injection from DM annihilations is effectively equivalent to have the smoother DM density profile suggested by $\gamma$ bounds [22].

Inverse Compton presently does not provide the dominant constraint, but it plays an important rôle. First because it directly probes if the $e^{ \pm}$excess is present in all the DM halo. As the energy spectrum of the $e^{ \pm}$excess is now strongly constrained by FERMI, HESS and PAMELA, the IC $\gamma$ flux can be reliably computed to be as in fig. 1c: all DM models able of fitting the $e^{ \pm}$excess 

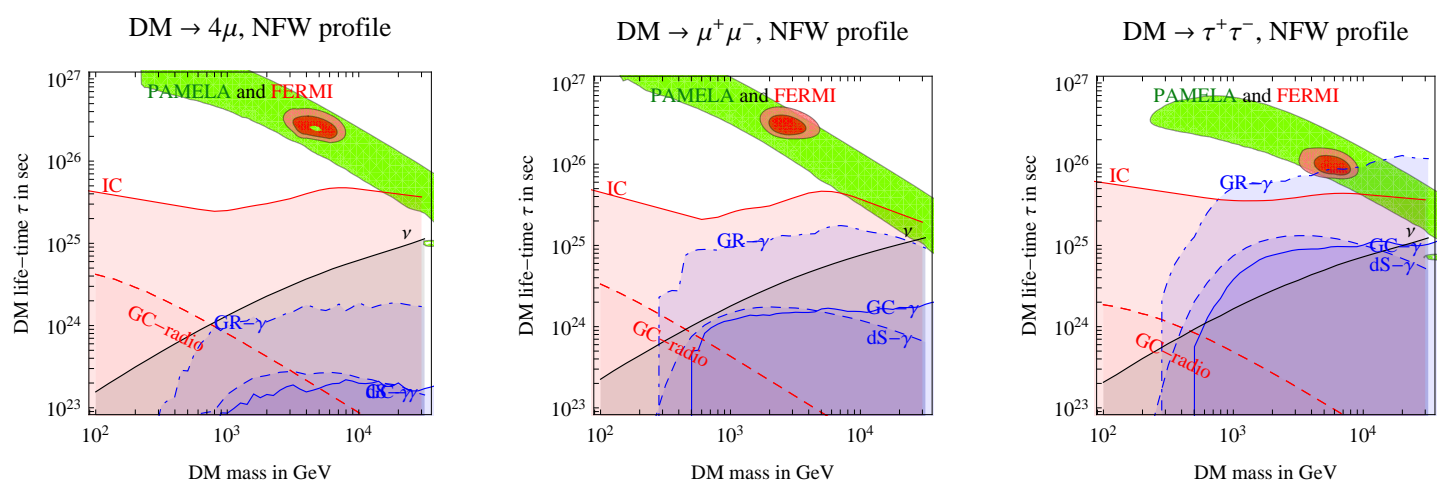

Figure 3: As in fig. 2, here for DM decaying into $\mu^{+} \mu^{-}$(middle), $\tau^{+} \tau^{-}$(right), $4 \mu$ (left).

predict roughly the same IC flux. Second, because so far FERMI $\gamma$ data have been released only in one angular region (with galactic latitude between $10^{\circ}$ and $20^{\circ}$, the region considered in fig. 1c) and only below $10 \mathrm{GeV}$ : fig. 1c shows that forthcoming FERMI data at higher energies should hopefully test the DM-related $\gamma$ excess.

Preliminary FERMI data [26] indicate a hint of an excess: a precise understanding of instrumental and astrophysical backgrounds is needed to hopefully reach a conclusion.

\subsection{Constraints from cosmology}

The DM annihilation rate, being proportional to the DM density squared, is enhanced in the early universe by the larger DM density, affecting cosmological observables in various stages of the universe history [23]:

1. DM annihilations at $T \sim \mathrm{MeV}$ affect $\mathrm{BBN}$ fragmenting ${ }^{4} \mathrm{He}, \mathrm{D},{ }^{3} \mathrm{He} .$. and thereby modifying their abundances. However, their primordial abundances are not safely measured.

2. DM annihilations at $T \lesssim \mathrm{eV}$ i.e. redshift $z \lesssim 1000$ reionize $\mathrm{H}$ after matter/radiation decoupling, such that $\gamma$ re-couple to matter, affecting the anisotropy pattern of CMB.

3. DM annihilations after structure formation, at $z \sim 10$, heat gas. However the effect depends on the unknown non-linear small-scale DM clustering.

All these constraints on $\sigma v$ are at the level of what needed by DM interpretations of the $e^{ \pm}$excesses. The second constraint is the strongest and most robust one, and it can be improved by the CMB measurements being performed by the PLANCK satellite.

\subsection{DM decay}

The alternative interpretation of the $e^{ \pm}$excesses in terms of DM decays solves three difficulties faced by DM annihilations. First, the decay rate is not linked with cosmological freeze-out, so that one does not need to invent Sommerfeld or other enhancements. Second, cosmological constraints 
do not apply, as the DM life-time is not enhanced in the early universe. Third, the decay rate is proportional to $\rho$ rather than to $\rho^{2}$ : thereby the $e^{ \pm}, \gamma$ injection term is less enhanced close to the Galactic Center where $\rho$ is large. Fig. 3 shows that, as a result, the DM decay interpretation of the $e^{ \pm}$excesses is compatible with $\gamma, v$ bounds even for a NFW DM density profile.

We see that the needed DM mass and lifetime are $M \sim 3 \mathrm{TeV}$ and $\tau \sim 10^{26} \mathrm{sec}$. DM that decays via a GUT-suppressed dimension 6 operator naturally give the needed $\tau \sim M_{\mathrm{GUT}}^{4} / M^{5}[24,25]$. Furthermore, if DM is a proton-like particle, composite of chiral fermions, with an asymmetry kept in thermal equilibrium by sphalerons down to the electroweak symmetry breaking scale at $T \sim 100 \mathrm{GeV}$, the cosmological DM abundance is naturally obtained as $\Omega_{\mathrm{DM}} / \Omega_{b} \sim e^{-M / T} M / m_{p}$ for $M \sim$ few TeV [24]. DM might be not the only particle charged under the new strong interactions: as well known the few $\mathrm{TeV}$ scale independently appears in technicolor solutions to the higgs mass hierarchy puzzle, where a strong $\lambda \sim 4 \pi$ coupling gives mass $M \sim 4 \pi v$ to chiral fermions.

\section{Conclusions}

The most plausible scenario for DM is that it is the relic of a new weak scale particle. If true, we should soon see direct and/or indirect signals of DM, and produce DM at the LHC.

Maybe we have already seen the first signals. The excesses in $e^{ \pm}$cosmic rays measured by PAMELA, FERMI (or ATIC) and HESS can be interpreted in terms of Dark Matter annihilations or decays into leptonic final states. The following solutions emerge:

- DM DM $\rightarrow \tau^{+} \tau^{-}$with $M \approx 3 \mathrm{TeV}$ provides one of the best fits to the $e^{ \pm}$spectra. However the $\tau$ decays into $\pi^{0}$ imply a large $\gamma$ flux, so that $\tau$ modes are compatible with bounds from $\gamma$ observations only if the (effective) DM density $\rho(r)$ is quasi-constant, a possibility disfavored by $N$-body simulations

- DM DM $\rightarrow V V \rightarrow \mu^{+} \mu^{-} \mu^{+} \mu^{-}$with $M \approx 3 \mathrm{TeV}$ provides one of the best fits to the $e^{ \pm}$ spectra. This solution is marginally compatible with $\gamma$ and $v$ bounds for the Einasto profile favored by $N$-body simulations: a more constant $\rho(r)$ is again preferred.

- DM that decays into $\tau^{+} \tau^{-}$or $\mu^{+} \mu^{-} \mu^{+} \mu^{-}$can fit the $e^{ \pm}$excesses compatibly with all $\gamma, v$ and cosmological constraints.

Other channels are less favored (e.g. $\mu^{+} \mu^{-}$and $e^{+} e^{-} e^{+} e^{-}$) or excluded $\left(e^{+} e^{-}\right)$.

If the $e^{ \pm}$excess is present everywhere in the DM halo (rather than only close to us, as if the excess is produced by a nearby pulsar), FERMI should be able to observe a related excess of $\gamma$ around $100 \mathrm{GeV}$, generated by Inverse Compton scattering of $e^{ \pm}$on galactic star-light and CMB.

The on-going analysis will have a huge impact on the Dark Matter field. Maybe the final result will be evidence for lepto-philic Dark Matter in $e^{ \pm}$and in $\gamma$. Or maybe that $e^{ \pm}$are polluted by extra astrophysical backgrounds, and DM did not show up in $\bar{p}$ nor in $\gamma$.

\section{References}

[1] WMAP collaboration, arXiv:astro-ph/0603449.

[2] For a recent summary see D.P. Finkbeiner, T. Lin, N. Weiner, arXiv:0906.0002. 
[3] B. Feldstein, A.L. Fitzpatrick, E. Katz, arXiv:0908.2991.

[4] PAMELA collaboration, arXiv:0810.4995.

[5] ATIC collaboration, Nature 456 (2008) 362.

[6] FERMI/LAT collaboration, arXiv:0905.0025.

[7] H.E.S.S. Collaboration, arXiv:0811.3894. H.E.S.S. Collaboration, arXiv:0905.0105.

[8] F. Donato, N. Fornengo, D. Maurin and P. Salati, Phys. Rev. D 69, 063501 (2004) [arXiv:astro-ph/0306207].

[9] M. Cirelli, M. Kadastik, M. Raidal, A. Strumia, Nucl. Phys. B813 (2009) 1 [arXiv:0809.2409].

[10] F. Donato, D. Maurin, P. Brun, T. Delahaye and P. Salati, Phys. Rev. Lett. 102 (2009) 071301 [arXiv:0810.5292].

[11] M. Ibe, H. Murayama and T. T. Yanagida, arXiv:0812.0072.

W. L. Guo and Y. L. Wu, Phys. Rev. D 79 (2009) 055012 [arXiv:0901.1450].

[12] A. Sommerfeld, Ann. Phys. 403 (1931) 257.

J. Hisano, S. Matsumoto and M. M. Nojiri, Phys. Rev. Lett. 92 (2004) 031303.

M. Cirelli, A. Strumia, M. Tamburini, Nucl. Phys. B787 (2007) 152 [arXiv:0706.4071].

[13] M. Cirelli, R. Franceschini, A. Strumia, Nucl. Phys. B800 (2008) 204 [arXiv:0802.3378] and ref.s therein.

[14] N. Arkani-Hamed, D. P. Finkbeiner, T. Slatyer and N. Weiner, arXiv:0810.0713.

[15] J.D. Bjorken, R. Essig, P. Schuster, N. Toro, arXiv:0906.0580.

[16] PAMELA collaboration, arXiv:0810.4994.

[17] T. Delahaye, R. Lineros, F. Donato, N. Fornengo and P. Salati, arXiv:0712.2312.

[18] P. Meade, M. Papucci, A. Strumia, T. Volansky, arXiv:0905.0480.

[19] P. Grajek, G. Kane, D. Phalen, A. Pierce and S. Watson, arXiv:0812.4555.

[20] G. Bertone, M. Cirelli, A. Strumia, M. Taoso, JCAP 0901 (43) 2009 [arXiv:0811.3744].

[21] J. Hisano, M. Kawasaki, K. Kohri and K. Nakayama, arXiv:0812.0219.

[22] I. Z. Rothstein, T. Schwetz and J. Zupan, arXiv:0903.3116.

[23] J. Hisano, M. Kawasaki, K. Kohri, T. Moroi and K. Nakayama, arXiv:0901.3582.

S. Galli, F. Iocco, G. Bertone and A. Melchiorri, Phys. Rev. D 80 (2009) 023505 [arXiv:0905.0003].

T. R. Slatyer, N. Padmanabhan, D.P. Finkbeiner, Phys. Rev. D 80 (2009) 043526 [arXiv:0906.1197].

G. Huetsi, A. Hektor and M. Raidal, arXiv:0906.4550.

M. Cirelli, F. Iocco and P. Panci, arXiv:0907.0719.

[24] E. Nardi, F. Sannino A. Strumia, JCAP 0903 (009) 2009 [arXiv:0811.4153].

[25] A. Arvanitaki et al., arXiv:0812.2075.

[26] In view of a contract with NASA, every photon detected by FERMI is publicly available at the web site fermi.gsfc.nasa.gov. I hope that collider physics will take advantage by such procedure, now common in astrophysics and cosmology, making all LHC data public. 